una estrategia de salud pública dirigida a reducir la mortalidad materna fue un elemento crítico para la obtención de buenos resultados.

En un período relativamente corto de tiempo se logró mejorar significativamente la oferta de APA en los programas nacionales. Estos resultados indican que el uso de la técnica de aspiración manual endouterina aumentó de $38 \%$ a $69 \%$ y la proporción de mujeres que acudieron al hospital por abortos incompletos y regresaron a sus hogares con algún método anticonceptivo aumento a más del doble.

En Guatemala falta mucho para lograr institucionalizar completamente la APA y disminuir el número de muertes maternas y de complicaciones, pero los resultados alcanzados hasta la fecha pueden ser útiles también en otros lugares. Se espera que la extensión de los programas de APA y la mejor comprensión del efecto de las complicaciones del aborto en la salud de las mujeres ayudarán a fortalecer el compromiso con estos programas y su sostenibilidad en el futuro. (Kestler E, et al. Scaling up post-abortion care in Guatemala: initial successes at national level. Reprod Health Matters. 2006;14:138-47.)

\section{La aplicabilidad de los principios de la promoción de la salud ante la amenaza de una pandemia}

Se sabe que hay una alta probabilidad de que ocurra una nueva pandemia de gripe en los próximos 10 años, pero con frecuencia se omite en el análisis el hecho de que la humanidad se encuentra en una situación histórica que le permite estar preparada.

Recientemente, la Escuela Nacional de Salud Pública (ENSP) de Lisboa, Portugal, acometió un proyecto dirigido a explorar las ventajas de enfrentar una pandemia de influenza con un enfoque innovador. En el proyecto se reconoce que la forma de concebir un evento es un factor determinante de cómo responder ante él. En consecuencia, ese proyecto ha concebido una posible pandemia de gripe como una nueva oportunidad para que las ciudades adopten un nuevo enfoque frente a este tipo de amenaza en el siglo XXI.

El enfoque descrito en el proyecto yuxtapone dos tipos de ciudades: ciudad susceptible y ciudad inteligente. Estas categorías demuestran cómo un enfoque centrado solamente en la situación médica y en la respuesta sanitaria (ciudad susceptible) puede cerrar la mente a innovaciones que pudieran ayudar a las ciudades y a sus habitantes más allá de una emergencia de salud pública (ciudad inteligente).
El consenso actual es que el aumento de la respuesta social - es decir, de las actividades que no son médicas - constituirá la principal medida que permitirá controlar una pandemia.

Los ciudadanos mismos son la mejor protección contra la propagación de la infección. En la ciudad inteligente, los ciudadanos se sienten parte interesada y un sentimiento de control sustituye en ellos a la sensación de miedo, impotencia e incertidumbre. Esto formará una sólida base para cualquier respuesta fructífera ante una emergencia y mejorará la calidad de vida de los ciudadanos en la medida en que sientan que ejercen control.

Todos los sectores del gobierno y de la sociedad deben participar en la preparación de medidas ante una pandemia mundial de gripe: lo mejor de la salud pública en el siglo XXI es su carácter dinámico y emprendedor, basado en la participación de comunidades bien coordinadas. Las organizaciones de la sociedad civil deben participar en este proceso desde su mismo inicio, si es posible.

En tiempos de crisis es necesario garantizar relaciones de confianza entre el sector empresarial, los servicios públicos - como el correo, el transporte público y las autoridades de orden interior- $-\mathrm{y}$, por supuesto, los servicios de salud. La ciudad inteligente construye estas relaciones mucho antes de que ocurra una crisis, en parte también porque esa estructura puede servir para muchas otras funciones cruciales de la organización y desarrollo de la ciudad.

La ciudad inteligente considera que saber prepararse para una pandemia es una forma de mejorar la calidad de vida en las ciudades contemporáneas: todo lo que se construya debe hacerse sobre la base de valores importantes para la comunidad. Tomando en cuenta que la detención de una epidemia en un país democrático se basa en el consenso social acerca de las principales acciones que se deben emprender, los ciudadanos deberán participar tanto como sea posible en el debate acerca de los valores, las metas y los enfoques que se utilizarán.

En la Conferencia sobre Promoción de Salud, celebrada en Bangkok, Tailandia en 2005, se insistió en que la promoción de la salud no es solo una estrategia para controlar las enfermedades crónicas y no trasmisibles, sino parte de la "nueva salud pública" que se requiere para poder enfrentar la amenaza de pandemias en la sociedad moderna. El empoderamiento de los ciudadanos a escala local para enfrentar esa amenaza constituye una tarea que exige inteligencia. (Kickbusch I. Flu City-Smart City: applying health promotion principles to a pandemic threat. Health Promot Intl. 2006; 21(2):85-7.) 